2005

\title{
Stochastic resonance of electrochemical aperiodic spike trains
}

\author{
P. Parmananda
}

Gerardo J. Escalera Santos

M. Rivera

Kenneth Showalter

Follow this and additional works at: https://researchrepository.wvu.edu/faculty_publications

\section{Digital Commons Citation}

Parmananda, P.; Escalera Santos, Gerardo J.; Rivera, M.; and Showalter, Kenneth, "Stochastic resonance of electrochemical aperiodic spike trains" (2005). Faculty Scholarship. 348.

https://researchrepository.wvu.edu/faculty_publications/348 


\title{
Stochastic resonance of electrochemical aperiodic spike trains
}

\author{
P. Parmananda, ${ }^{1}$ Gerardo J. Escalera Santos, ${ }^{1}$ M. Rivera, ${ }^{1}$ and Kenneth Showalter ${ }^{2}$ \\ ${ }^{1}$ Facultad de Ciencias, UAEM, Avenida Universidad 1001, Colonia Chamilpa, Cuernavaca, Morelos, Mexico \\ ${ }^{2}$ Department of Chemistry, West Virginia University, Morgantown, West Virginia 26506-6045, USA \\ (Received 3 September 2004; revised manuscript received 11 November 2004; published 23 March 2005)
}

\begin{abstract}
Aperiodic stochastic resonance in an electrochemical system with excitable dynamics is characterized in experiments and simulations. Two different spike trains, one with stochastic and the other with chaotic interspike intervals, are imposed on the system as subthreshold aperiodic signals. Information transmission is quantified by the cross correlation between the subthreshold input signal and the noise induced system response. A maximum is exhibited in the input-output correlation as a function of the noise amplitude. Numerical simulations with an electrochemical model are in excellent agreement with the experimental observations.
\end{abstract}

DOI: 10.1103/PhysRevE.71.031110

PACS number(s): 05.40.- a, 05.45.Xt

A subthreshold signal that is otherwise undetectable can often be detected in the presence of noise, and typically there is an optimal noise level for information transmission. Most studies of this phenomenon, known as stochastic resonance (SR), have been on the noise enhanced system response to subthreshold periodic signals [1-4]. SR has been studied in a wide variety of physical, chemical, and biological systems [5-9] and has been proposed as a means for improving signal detection and enhancing information transfer [6-12]. A related phenomenon is coherence resonance (CR) [13-16], where the system itself gives rise to the noise induced response rather than a subthreshold signal. Both SR and CR are generic system responses to noise and consequently are found in a wide variety of dynamical systems.

While studies of SR typically focus on periodic subthreshold signals, some investigations have examined more complex signals such as aperiodic signals [17-19]. As in SR, there is an optimal noise level for information transfer in aperiodic stochastic resonance (ASR) [18]. The fidelity between the aperiodic input stimulus and the system response can be quantified by determining the cross correlation coefficient (power norm) $[18,20]$. ASR has been studied theoretically $[18,20-22]$ and has been characterized in several experimental studies [23-25]. In this paper, we present a characterization of ASR in a chemical system exhibiting excitable dynamics. Moreover, we expand the phenomenon of ASR by characterizing the noise induced system response to subthreshold spike trains with aperiodic interspike intervals.

The experimental system comprised of a three-electrode electrochemical cell configured for studying the potentiostatic (EG\&G PARC model 362 potentiostat) electrodissolution of iron in a solution of copper sulfate and sulfuric acid. The anode was an iron disk (5.0 mm diameter) embedded in epoxy, which was exposed to the solution at a polished crosssection surface. The electrolyte solution was a mixture of 1.0 $\mathrm{M}$ sulfuric acid and $0.4 \mathrm{M}$ copper sulfate. The anodic potential $V$, measured relative to a saturated calomel electrode (SCE), was used as the control parameter. The cathode was a copper rod, $5.0 \mathrm{~mm}$ in diameter and $2 \mathrm{~cm}$ long, immersed in the solution. Oscillations in the anodic current $I$ (the current between the anode and the cathode) were recorded using a 12-bit data acquisition card at a sampling frequency of 33 Hz. More details regarding the experimental setup along with a schematic of the electrochemical cell can be found in our previously published work [26]. The imposed external noise was produced using a random number generator, with the output distributed over the interval $[-1,1]$ and corresponding to white noise with a Gaussian distribution. It needs to be pointed out that for the purposes of our experiments, the Gaussian distribution of the superimposed noise was normalized such that its output is restricted between the interval $[-1,1]$. The protocol used for the normalization of the superimposed noise is as follows: A finite time series of noise with a Gaussian distribution (zero mean and standard deviation one) was generated numerically. Subsequently, this noise time series was confined to the interval $[-1,1]$ by dividing all the noise values by the maximum absolute number present in this finite time series. Consequently the altered noise series is bounded in the desired interval of $[-1,1]$ and was converted to an analog signal. This analog signal was thereafter combined (additively) with the anodic voltage via the potentiostat. The noise amplitude was varied with a frequency of approximately $1.25 \mathrm{~Hz}$.

Before studying the noise induced dynamics, we characterized the behavior of the autonomous system. Different dynamical responses of the anodic current $I$ were observed as a function of the anodic voltage $V$, the bifurcation parameter [27]. Two basic types of dynamics were observed: stationary state behavior, with a constant current response, and period-1 oscillations emerging from a supercritical Hopf bifurcation, where $V_{H} \approx 175 \mathrm{mV}$ (not shown). At anodic voltages slightly above the Hopf bifurcation, small, sinusoidal oscillations were observed. At higher voltages, relaxation oscillations were exhibited in which the period increased with an increase in voltage. This increase in period as a function of the bifurcation parameter, shown in Fig. 1, is characteristic of a homoclinic bifurcation, such as a saddle-node or a saddleloop bifurcation. Our previous study of this system [26] demonstrated that period lengthening occurs until oscillations cease at the homoclinic bifurcation point $V_{h c}$.

For our experiments on aperiodic stochastic resonance, the reference voltage $V_{0}$ was chosen so that $V_{0}>V_{h c}$ and excitable steady state behavior was exhibited. The anodic 


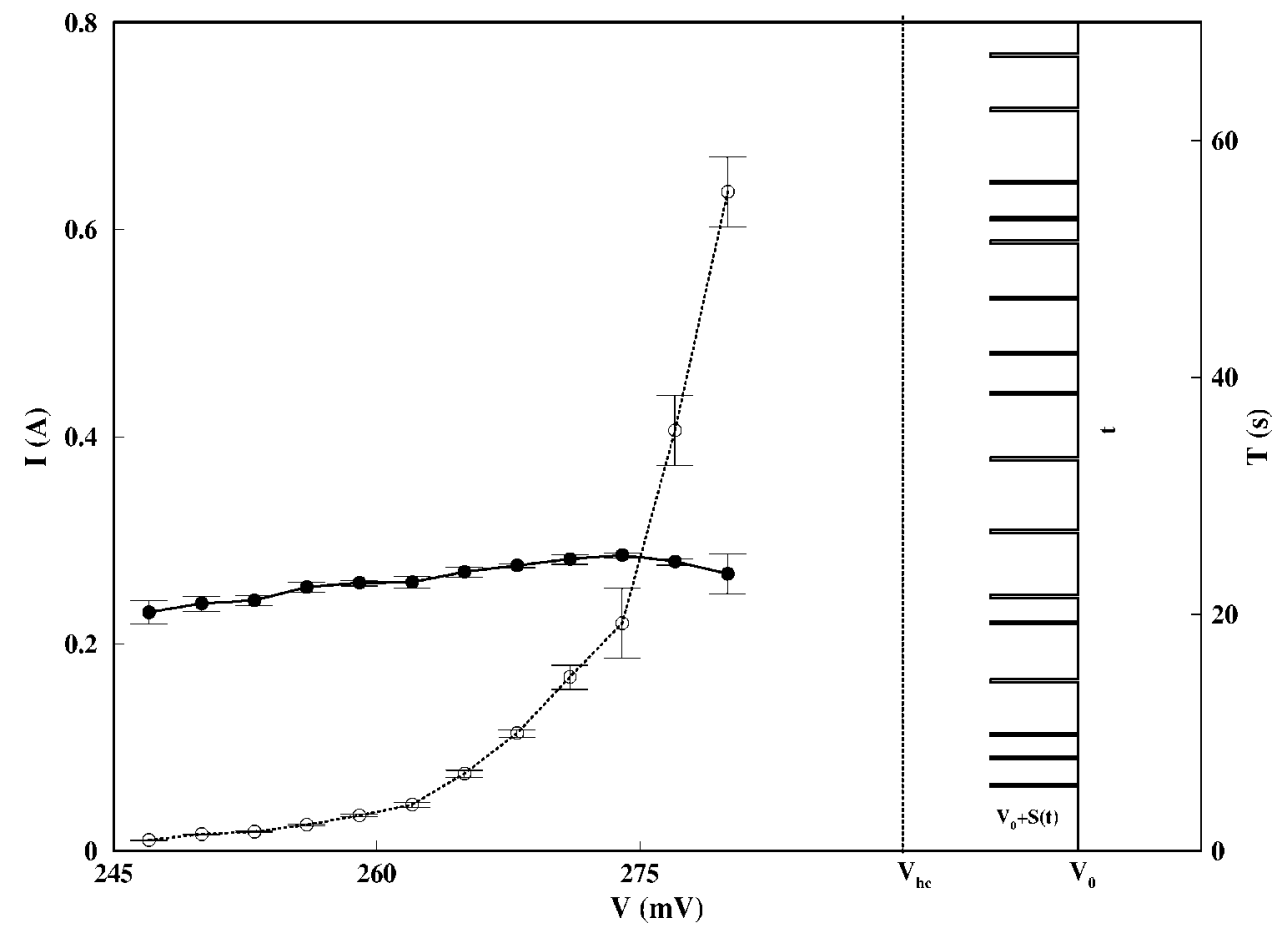

FIG. 1. Amplitude $I$ (filled circles) and period $T$ (empty circles) of oscillation in the anodic current as a function of the anodic voltage $V$. The exponential increase in period and relatively constant amplitude of oscillation indicates a homoclinic bifurcation.

voltage $V$ was then defined as $V=V_{0}+S(t)+D \xi$, where $V_{0}$ and the subthreshold aperiodic pulse train $S(t)$ were set such that $V=V_{0}+S(t)>V_{h c}$, i.e., the subthreshold signal never perturbed the system into the oscillatory regime. The amplitude of the imposed Gaussian white noise $\xi$ is $D$. We studied the system response as a function of the noise amplitude $D$.

To quantify the information transfer, we use the power norm, defined by [20]
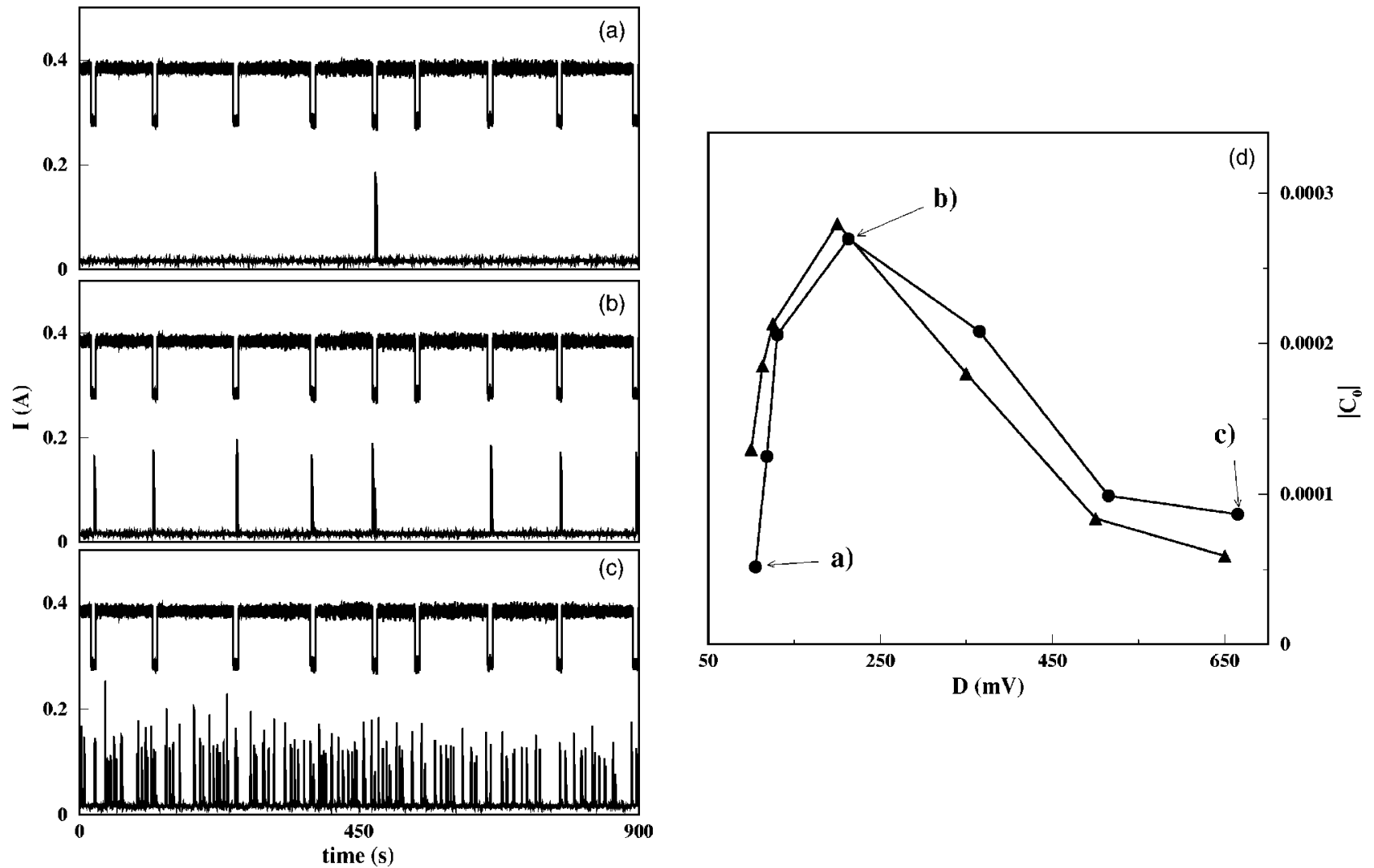

FIG. 2. Noise induced system response in the anodic current $I$ for low (a), medium (b), and high (c) amplitude noise from a subthreshold aperiodic signal with stochastic interspike intervals. Panel (d) shows the cross correlation $\left|C_{0}\right|$ as a function of noise level for two series of experiments (circles and triangles) with the same reference voltage $V_{0}=450 \mathrm{mV}$. 


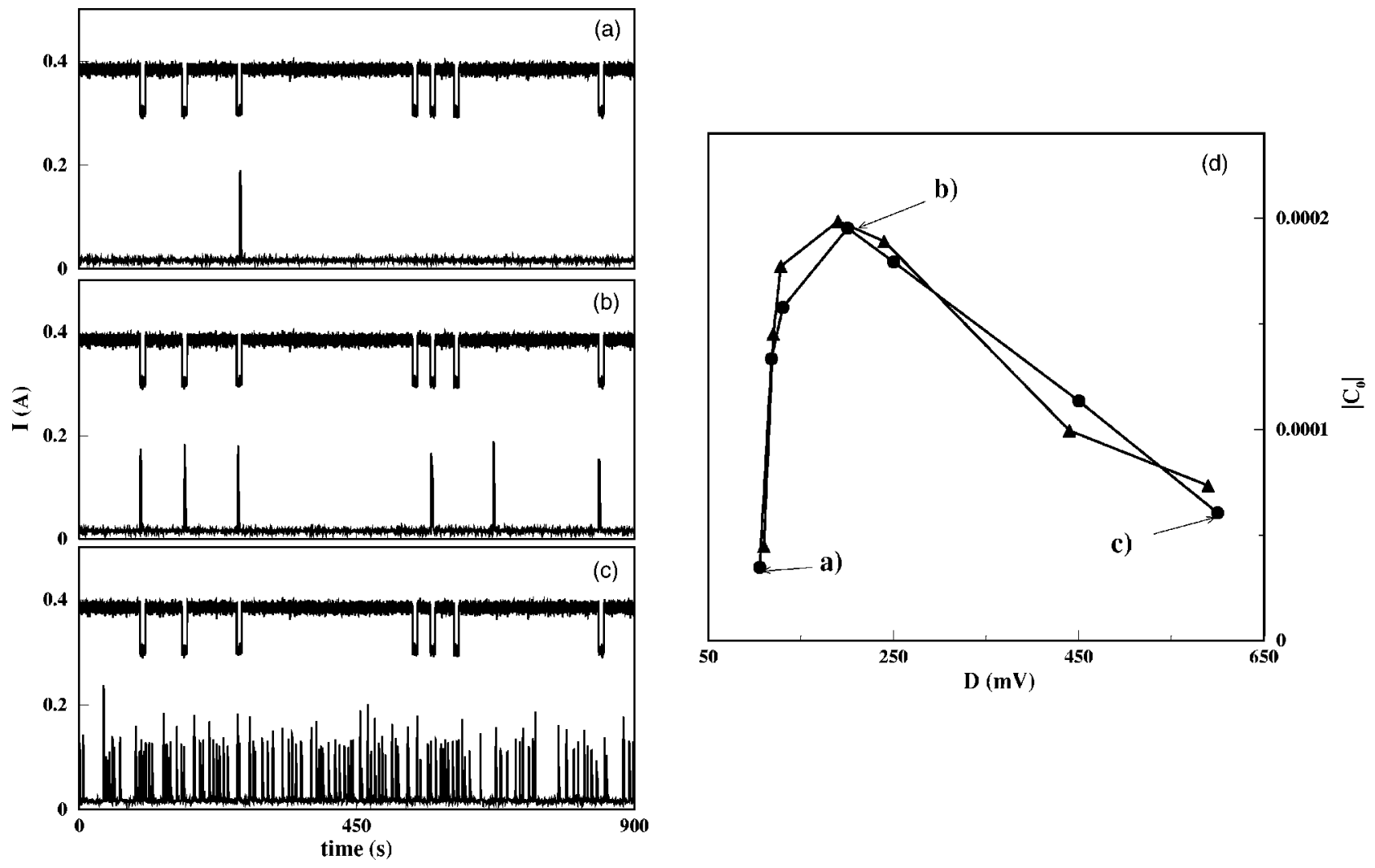

FIG. 3. Noise induced system response in the anodic current $I$ for low (a), medium (b), and high (c) amplitude noise for a subthreshold aperiodic signal from a deterministic model exhibiting chaotic interspike intervals. Panel (d) shows the cross correlation $\left|C_{0}\right|$ as a function of noise level for two series of experiments (circles and triangles) with the same reference voltage $V_{0}=450 \mathrm{mV}$.

$$
C_{0}=\left\langle\left(x_{1}-\left\langle x_{1}\right\rangle_{t}\right)\left(x_{2}-\left\langle x_{2}\right\rangle_{t}\right)\right\rangle_{t},
$$

where $x_{1}$ represents the time series of the aperiodic input signal, $x_{2}$ represents the noise induced response of the electrochemical system, and \langle\rangle$_{t}$ denotes the respective time averages. The power norm is a measure of the coincidence fidelity between the subthreshold aperiodic input signal and the noise induced system response.

In our first set of experiments, the subthreshold aperiodic pulse train was constructed by using a random number generator (ran1) [28] that yields uniform random deviates between the interval $[0,1]$. These numbers were subsequently rescaled to provide appropriate interspike intervals suitable for our experiments. The pulse amplitude was chosen to be $-100 \mathrm{mV}$ and the pulse duration $8.0 \mathrm{~s}$. Thus, while the amplitude and pulse duration remain constant, the interspike interval varies stochastically. Figures 2(a)-2(c) show time series of the system response (lower curves) with identical subthreshold spike trains (upper curves) for three different amplitudes of imposed noise. We see in panel (a) that there is little correspondence between the subthreshold signal and the system response at a low noise amplitude, while there is excellent correspondence at an intermediate noise amplitude, panel (b). In panel (c), the subthreshold signal is lost in the system response to the high amplitude noise. Figure 2(d) shows the power norm $\left|C_{0}\right|$ as a function of the noise amplitude $D$ for two experimental runs with identical reference voltages $V_{0}$. The two curves exhibit a unimodal structure, where the maximum corresponds to the optimal noise level for maximum information transfer.

In our second set of experiments, we used a deterministic model that simulates irregular neural spiking [29] as the subthreshold aperiodic signal. The spikes of the chaotic time series have a relatively constant amplitude of approximately $100 \mathrm{mV}$. Although the origin of the irregular spiking is purely deterministic, the histogram for the spike sequence as shown in Fig. 3(a) from the work of Baier et al. [29] seems to fit well with a Poisson distribution [29]. We again imposed noise with a successively higher amplitude $D$ in a sequence of experiments and measured the system response, with the same imposed subthreshold aperiodic spike train and reference voltage $V_{0}$. Figures 3(a)-3(c) show the stimulusresponse time series for low (a), medium (b), and high (c) amplitude noise. Again we see that there is an intermediate noise amplitude, panel (b), that gives rise to an excellent stimulus-response correspondence, while lower and higher amplitude noise produces little correspondence. As in Fig. 2, the upper trace in each panel corresponds to the subthreshold aperiodic spike train and the lower trace corresponds to the system response due to the imposed noise. Figure 3(d) shows $\left|C_{0}\right|$ as a function of $D$, quantifying the input-output correlation for two experimental runs with the same reference voltage $V_{0}$. As in the earlier experiments with a subthreshold stochastic signal, the power norm exhibits a maximum in each experiment that corresponds to the maximum information transfer. 


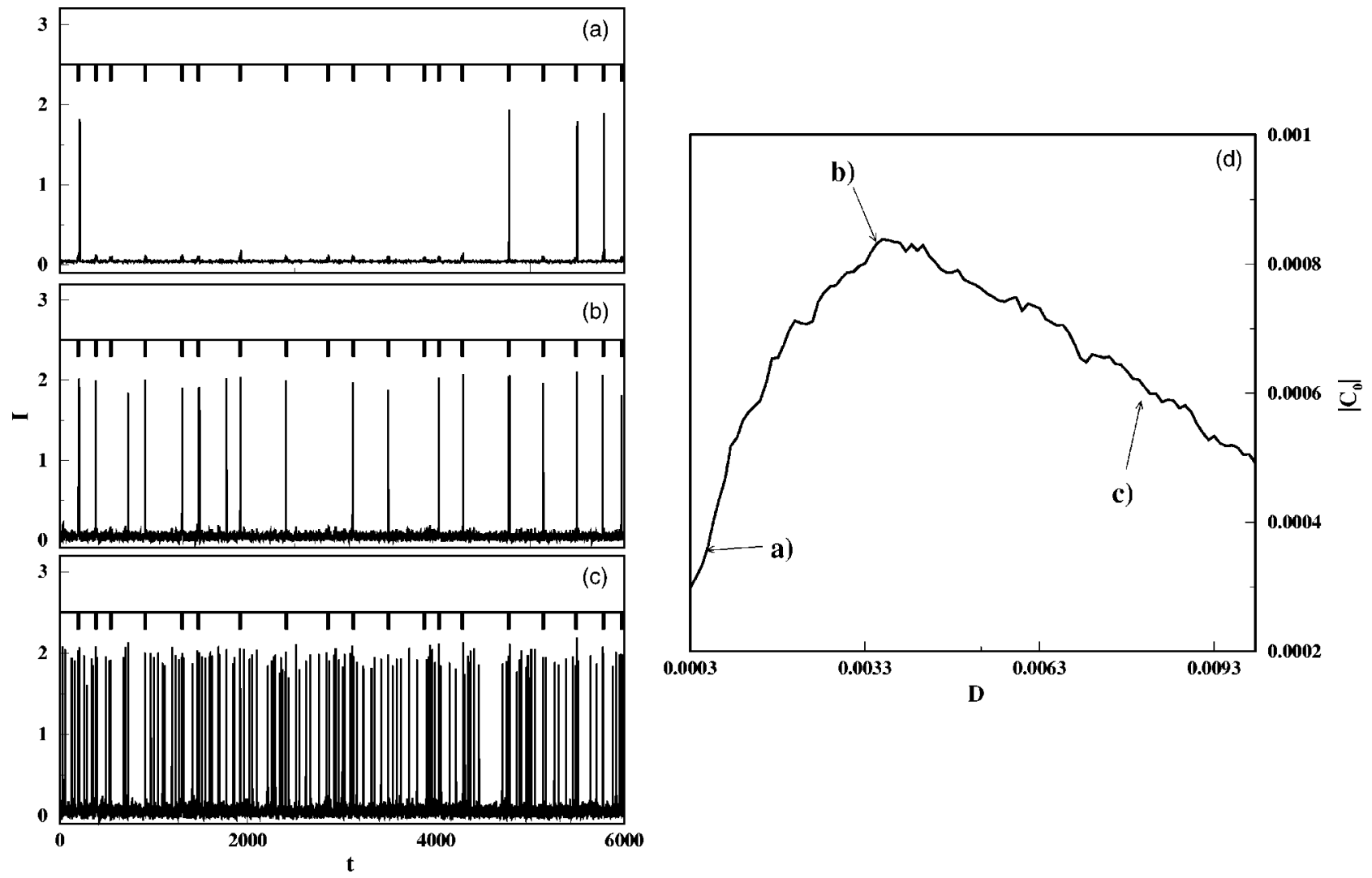

FIG. 4. Numerically computed noise induced system response in the anodic current $I$ for low (a), medium (b), and high (c) amplitude noise for a subthreshold aperiodic signal with stochastic interspike intervals. Panel (d) shows the cross correlation $\left|C_{0}\right|$ as a function of noise level for a simulation run with a reference voltage $v_{0}=29.34$. All the quantities plotted are dimensionless.

To corroborate our experimental measurements, we carried out numerical simulations using a two-dimensional model developed to simulate the dynamics of an electrochemical cell under potentiostatic conditions [30]:

$$
\begin{gathered}
\epsilon \dot{u}=\frac{v-u}{R}-f(u, c), \\
\dot{c}=\frac{u-v}{R}+(1-c)+\alpha f(u, c),
\end{gathered}
$$

where

$$
f(u, c)=c\left(a_{1} u+a_{2} u^{2}+a_{3} u^{3}\right) .
$$

The variables $u(t)$ and $c(t)$ correspond to the electrode potential and the surface concentration, respectively. The three system parameters, $\epsilon, R$, and $v$, represent the double layer capacitance, the Ohmic resistance, and the applied potential, respectively. Equation (2) represents the conservation of charge, and Eq. (3) describes the mass balance. The anodic current $I$ is the observable [defined as $(v-u) / R$ ], corresponding to our experimental measurements. The model system and its bifurcation structure have been characterized by Karantonis et al. [30]. Dynamical behavior much like we observed in our experiments is found for the following values of the parameters: $\quad \epsilon=0.03, \alpha=0.1, R=10, a_{1}$ $=1.125, a_{2}=-0.075$, and $a_{3}=0.00125$. The anodic voltage $v$, the bifurcation parameter, was varied to map out different responses of the model.

For anodic voltage values $28.097 \leqslant v \leqslant 29.235$, limit cycle behavior is displayed that gives way to stationary state behavior for $v>29.235$. As in the experiments, the value of the anodic voltage $v$ was chosen such that the autonomous system exhibited excitable steady state behavior. The applied potential $v$ was then perturbed by external perturbations in which $v=v_{0}+S(t)+v_{0} D \xi$, where the reference voltage $v_{0}$ and the subthreshold aperiodic signal $S(t)$ are set so that $v=v_{0}$ $+S(t)$ remains in the nonoscillatory regime. The amplitude of the superimposed noise is $D$ and $\xi$ represents the imposed Gaussian white noise with zero mean and unit standard deviation. The differential equations of model (2) and (3) were integrated using the Euler-Maruyama algorithm [31], specifically designed for solving stochastic differential equations.

As in our experiments, we first consider a subthreshold aperiodic spike train constructed with a stochastic time interval between successive pulses. The pulse amplitude and width were chosen to be -0.1 and 20 , respectively, in dimensionless units. The time series in Figs. 4(a)-4(c) display behavior much like that shown in Fig. 2, where the best correspondence between the input and output occurs at an intermediate noise amplitude. We also characterized the information transfer by calculating the power norm $\left|C_{0}\right|$. Figure 4(d) shows $\left|C_{0}\right|$ as a function of noise amplitude $D$ for a given reference voltage where the autonomous system exhibits excitable steady state behavior. 


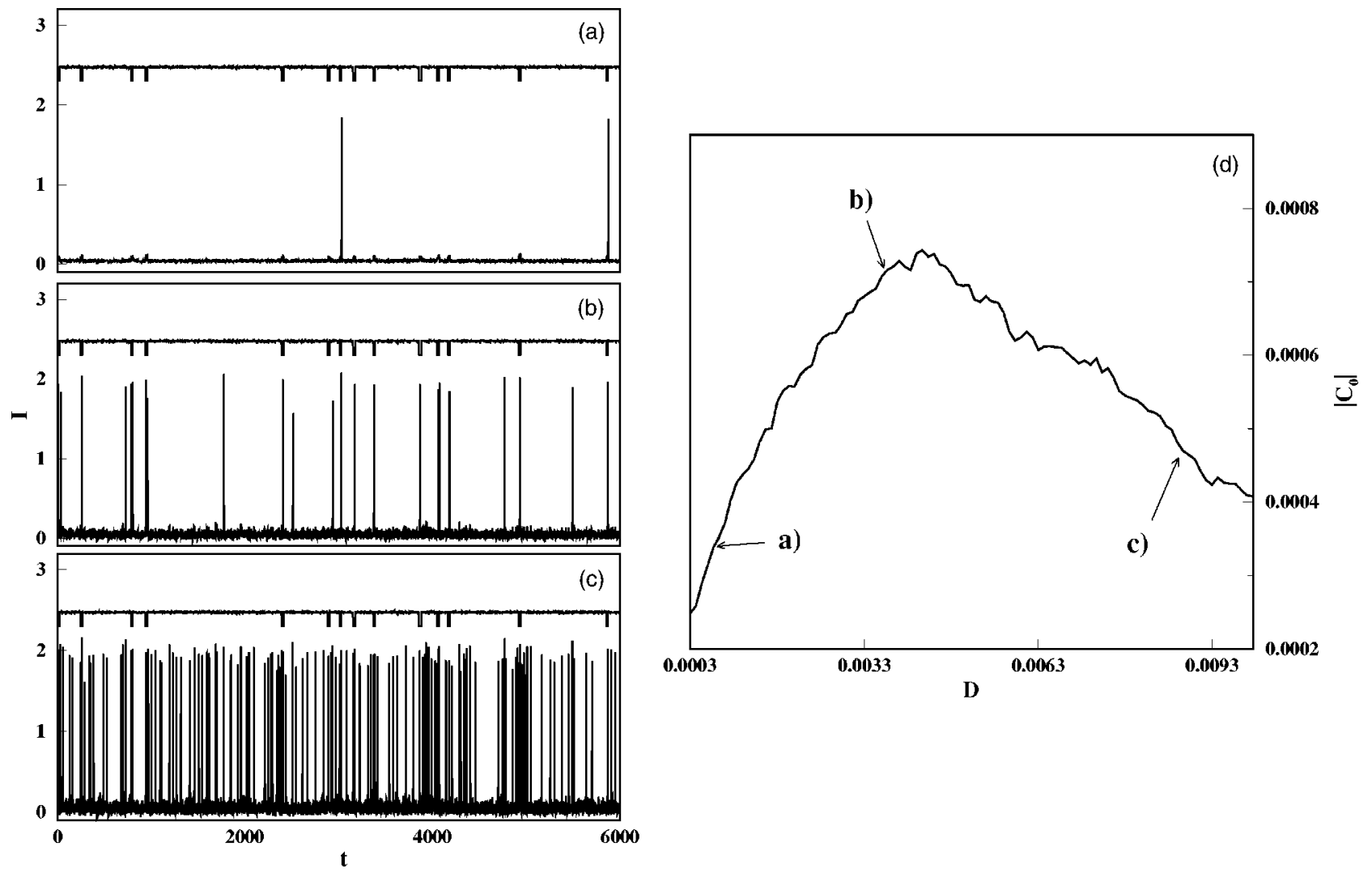

FIG. 5. Numerically computed noise induced system response in the anodic current $I$ (dimensionless units) for low (a), medium (b), and high (c) amplitude noise for subthreshold aperiodic signals from a deterministic model exhibiting chaotic interspike intervals. Panel (d) shows the cross correlation $\left|C_{0}\right|$ as a function of noise level for a simulation run with a reference voltage $v_{0}=29.34$. All the quantities plotted are dimensionless.

We next consider an aperiodic pulse train from the model for irregular neural spiking as the subthreshold signal [29], where the reference voltage was chosen such that the autonomous system exhibits an excitable steady state. Figure 5 shows three time series for low (a), medium (b), and high (c) amplitude noise. The upper trace again corresponds to the subthreshold spike train, while the lower trace corresponds to the noise induced system response. Panel (d) shows the power norm $\left|C_{0}\right|$ as a function of the noise amplitude $D$. As in the experimental results in Fig. 3, the maximum information transfer between the subthreshold signal and the noise induced system response occurs at an optimum amplitude of the imposed noise.

Our study demonstrates that SR occurs for aperiodic subthreshold signals in electrochemical systems. The ASR in our system differs from that in previous studies in that the aperiodicity occurs in the interspike interval, rather than in the amplitude, in both the stochastic and the chaotic systems. In neurophysiological sensory systems, the stimuli are often comprised of aperiodic spike trains, and ASR could provide a means for enhanced information transmission in these noisy systems. In addition, we have characterized ASR in an excitable electrochemical system that occurs near a homoclinic infinite-period bifurcation. While our numerical studies show excellent agreement with the experimentally measured ASR, it is important to point out that, for the parameters chosen here, the electrochemical model system does not exhibit a homoclinic bifurcation. This suggests that the nature of the bifurcation is perhaps not of essential importance in spike train ASR. We hope that this and future studies of SR with aperiodic spike trains will provide insights into the dynamics of noisy neuronal systems.

P.P. thanks CONACyT for supporting this research, and K.S. thanks the National Science Foundation (Grant No. CHE-0415392) and the Office of Naval Research (Contract No. N00014-04-1-0730). 
[1] R. Benzi, A. Sutera, and A. Vulpiani, J. Phys. A 14, L453 (1981).

[2] R. Benzi, G. Parisi, A. Sutera, and A. Vulpiani, Tellus 34, 10 (1982).

[3] C. Nicolis and G. Nicolis, Tellus 33, 225 (1981); C. Nicolis, ibid. 34, 1 (1982).

[4] L. Gammaitoni, P. Hanggi, P. Jung, and F. Marchesoni, Rev. Mod. Phys. 70, 223 (1998).

[5] F. Moss, A. Bulsara, and M. F. Shlesinger, J. Stat. Phys. 70, 1 (1993).

[6] A. Longtin, A. Bulsara, and F. Moss, Phys. Rev. Lett. 67, 656 (1991)

[7] A. Föster, M. Merget, and F. W. Schneider, J. Phys. Chem. 100, 4442 (1996).

[8] T. Amemiya, T. Ohmori, M. Nakaiawa, and T. Yamaguchi, J. Phys. Chem. 102, 4537 (1998).

[9] G. J. Escalera Santos and P. Parmananda, Phys. Rev. E 65, 067203 (2002)

[10] P. Hanggi, ChemPhysChem 3, 285 (2002).

[11] B. Lindner, A. Longtin, and A. Bulsara, Neural Comput. 15, 1761 (2003).

[12] B. Lindner, L. Schimansky-Geier, and A. Longtin, Phys. Rev. E 66, 031916 (2002).

[13] G. Giacomelli, M. Giudici, S. Balle, and J. R. Tredicce, Phys. Rev. Lett. 84, 3298 (2000).

[14] Hu Gang, T. Ditzinger, C. Z. Ning, and H. Haken, Phys. Rev. Lett. 71, 807 (1993).

[15] A. Pikovsky and J. Kurths, Phys. Rev. Lett. 78, 775 (1997).

[16] I. Z. Kiss, J. L. Hudson, G. J. Escalera Santos, and P. Parmananda, Phys. Rev. E 67, 035201 (2003).
[17] Hu Gang, G. De-chun, W. Xiao-dong, Y. Chun-yuan, Q. Guang-rong, and L. Rong, Phys. Rev. A 46, 3250 (1992).

[18] J. J. Collins, C. C. Chow, and T. T. Imhoff, Phys. Rev. E 52, R3321 (1995).

[19] J. J. Collins, C. C. Chow, A. C. Capela, and T. T. Imhoff, Phys. Rev. E 54, 5575 (1996).

[20] C. Eichwald and J. Walleczek, Phys. Rev. E 55, R6315 (1997).

[21] G. C. Kember, G. A. Fenton, K. Collier, and J. A. Armour, Phys. Rev. E 61, 1816 (2000).

[22] D. Rousseau, J. Rojas Varela, and F. Chapeau-Blondeau, Phys. Rev. E 67, 021102 (2003).

[23] J. J. Collins, T. T. Imhoff, and P. J. Grigg, J. Neurophysiol. 76, 642 (1996).

[24] S. Barbay, G. Giacomelli, and F. Marin, Phys. Rev. Lett. 85, 4652 (2000).

[25] S. Barbay, G. Giacomelli, and F. Marin, Phys. Rev. E 63, 051110 (2001).

[26] G. J. Escalera Santos, M. Rivera, M. Eiswirth, and P. Parmananda, Phys. Rev. E 70, 021103 (2004).

[27] A. Karantonis, Y. Miyakita, and S. Nakabayashi, Phys. Rev. E 65, 046213 (2002).

[28] Numerical Recipes in Fortran, second edition (Cambridge University Press, Cambridge, England, 1992).

[29] G. Baier, G. J. Escalera Santos, H. Perales, M. Rivera, M. Müller, R. Leder, and P. Parmananda, Phys. Rev. E 62, R7579 (2001).

[30] A. Karantonis and S. Nakabayash, Chem. Phys. Lett. 347, 133 (2001).

[31] D. J. Higham, SIAM Rev. 43, 525 (2001). 